\title{
Effect of surface roughness on the anomalous Hall effect in Fe thin films
}

\author{
Qiang Zhang $\odot,{ }^{1, *}$ Dongxing Zheng, ${ }^{1}$ Yan Wen, ${ }^{1}$ Yuelei Zhao, ${ }^{1}$ Wenbo Mi $\odot,{ }^{2}$ Aurelien Manchon, ${ }^{1}$ \\ Olivier Boulle, ${ }^{3}$ and Xixiang Zhang $\oplus^{1, \dagger}$ \\ ${ }^{1}$ King Abdullah University of Science and Technology (KAUST), Physical Science and Engineering Division (PSE), \\ Thuwal 23955-6900, Saudi Arabia \\ ${ }^{2}$ Tianjin Key Laboratory of Low Dimensional Materials Physics and Preparation Technology, School of Science, \\ Tianjin University, Tianjin 300354, China \\ ${ }^{3}$ IRIG-SPINTEC, CNRS, CEA, Grenoble INP, Université Grenoble Alpes, 38000 Grenoble, France
}

(Received 4 September 2019; revised manuscript received 13 March 2020; accepted 17 March 2020; published $\mathrm{xxxxxx)}$

\begin{abstract}
Surface roughness plays an important role on the magnetotransport properties of thin films, especially in ultrathin films. In this work, we prepared Fe thin films with various surface roughness by using different seed layers and studied the electrical transport and anomalous Hall effect. By tuning surface roughness scattering, the longitudinal resistivity $\left(\rho_{x x}\right)$ measured at $5 \mathrm{~K}$ increases by one order of magnitude and the corresponding anomalous Hall resistivity $\left(\rho_{\mathrm{AHE}}\right)$ increases by three times with increasing roughness. The intrinsic, skewscattering, and side-jump contributions to $\rho_{\mathrm{AHE}}$ were separated from our data. The anomalous Hall angle depends on the surface roughness, which may be of importance to the material engineering for achieving large spin Hall angle.
\end{abstract}

DOI: 10.1103/PhysRevB.00.004400

\section{INTRODUCTION}

Anomalous Hall effect (AHE) in magnetic materials, since its discovery [1], has been extensively studied for the fundamental interests and potential applications. It is generally accepted that the intrinsic and extrinsic mechanisms, both of which are related to spin-orbit coupling, are responsible for the AHE. Microscopically, the intrinsic mechanism [2-5], independent of impurity scattering, originates from the Berry phase of the occupied Bloch states. The extrinsic mechanism arising from impurity scattering includes two contributions, skew scattering [6], and side jump [7]. Skew scattering comes from the asymmetric scattering at impurity sites and side jump emerges from transverse displacement of the wave function by impurity scattering.

Theories also suggested the scaling relations $[2,6,7]$ between anomalous Hall resistivity $\left(\rho_{\mathrm{AHE}}\right)$ and longitudinal resistivity $\left(\rho_{x x}\right)$ for each mechanism: $\rho_{\mathrm{AHE}} \propto \rho_{x x}^{2}$ for both the intrinsic and extrinsic side-jump mechanism, while $\rho_{\mathrm{AHE}} \propto \rho_{x x}$ for extrinsic skew-scattering mechanism. The scaling law is usually applied to experimental data to explore the underlying mechanisms. Experimentally, both the $\rho_{\mathrm{AHE}}$ and $\rho_{x x}$ should be tuned in some range to give a reliable scaling relation. To this end, one option is varying the film thickness where the $\rho_{x x}$ could be changed by surface scattering [8-12]. Films with different thickness are also eligible to study the surface scattering effect on the AHE $[13,14]$. However, surface scattering strength/potential is not only related to the thickness of the

${ }^{*}$ Current address: Core Technology Platforms, New York University Abu Dhabi, P.O. Box 129188, Abu Dhabi, United Arab Emirates.

†Corresponding author: xixiang.zhang@kaust.edu.sa films but also to the surface roughness, especially in ultrathin films. Furthermore, the studies of spintronic topics of spin orbital torque (SOT), interfacial Dzyaloshinskii-Moriya Interaction (DMI) and perpendicular magnetic anisotropy (PMA) are all involved with ultrathin films. The surface/interface roughness may play an important role on these topics (see Sec. 1 in Ref. [15]), which has been omitted in these studies. Few theoretical studies have suggested that surface roughness scattering could lead to a completely different scaling relation between $\rho_{\text {AHE }}$ and $\rho_{x x}$ for spin Hall effect [16] or enhance the spin Hall angle [17] in ultrathin films. However, an experimental study of surface roughness effect on magnetotransport properties, particularly AHE, in thin films is still lacking.

In this study, we report the surface roughness effect on the AHE in Fe thin films (see the definition of surface roughness in Sec. 2 of Ref. [15]). The surface roughness could be affected by several factors in deposited thin films: seed layer on substrate [18], substrate temperature [19], thickness of deposited films [20], argon pressure during deposition [21], etc. To study the surface roughness effect, we must keep all other parameters of the samples the same and only vary the surface roughness. Therefore, we cannot use different deposition temperature or thickness of films since it will change the microstructure or surface scattering strength. We then used different materials of seed layers to tune the surface roughness. This idea is utilizing the wetting property of materials, which is similar to the chemistry concept of the wettability of liquid contacting with a solid surface. By this method, we may most probably be able to keep all parameters (such as microstructure, thickness, etc.) the same but the roughness. It is, therefore, feasible to the study of the impact of surface roughness scattering on the AHE. 


\section{EXPERIMENTAL METHODS}

The samples were deposited on substrates of oxidized silicon wafers by sputtering system (Rotaris, Singulus). The structure of the films is, from the substrate side, seed layer $(0.2 \mathrm{~nm}) / \mathrm{Fe}(5 \mathrm{~nm}) / \mathrm{SiO}_{2}(5 \mathrm{~nm})$ with different seed layers ( Ta, $\mathrm{Ir}_{20} \mathrm{Mn}_{80}, \mathrm{MgO}, \mathrm{Ru}, \mathrm{Co}_{60} \mathrm{Fe}_{20} \mathrm{~B}_{20}, \mathrm{SiO}_{2}, \mathrm{Co}, \mathrm{Ni}, \mathrm{CuN}$, $\mathrm{Cu})$. The samples were deposited, at room temperature, with the base pressure lower than $8.0 \times 10^{-9}$ mbar and the process pressure $3 \times 10^{-3}$ mbar. The deposition of $\mathrm{SiO}_{2}$ with $5 \mathrm{~nm}$ thickness as a top layer for each sample is to prevent sample oxidization. The surface roughness of the samples was characterized by atomic force microscope (AFM) (Dimension Icon, Bruker). The cross-sections of the samples were imaged by high-resolution transmission electron microscopy (HRTEM) (Titan 80-300, FEI). Grazing incidence diffraction (GID) measurements were carried out using x-ray diffraction (XRD) (D8 ADVANCE DAVINCI design, Bruker) with $\mathrm{Cu}$ $K \alpha$ radiation. The longitudinal resistivity and Hall resistivity were measured by a physical property measurement system (Dynacool 14T, Quantum Design). The Hall-bar samples with dimensions $1000 \mu \mathrm{m}$ (length) $\times 50 \mu \mathrm{m}$ (width) were patterned by photolithography and ion beam etching for transport measurements. A five-contact geometry was used for simultaneously measuring the Hall resistivity and longitudinal resistivity on the same piece of sample. A DC constant current of $20 \mu \mathrm{A}$ was applied during all electrical transport measurements. The sample electrodes were connected to the sample holder of the PPMS by wire bonder using aluminum wires.

\section{RESULTS AND DISCUSSION}

\section{A. Structural characterizations}

To demonstrate the surface roughness of the Fe layers, the samples was imaged by AFM. Due to the existence of the protection layers, the AFM images only give the surface roughness of the $\mathrm{SiO}_{2}$ layers, which could indirectly reflect the surface roughness of Fe layers. As seen in Fig. S1 and the corresponding analysis in Supplemental Material [15], the roughness of sample $\mathrm{Cu} / \mathrm{Fe}$ is higher than that of sample $\mathrm{Ta} / \mathrm{Fe}$, which indicates the larger roughness of $\mathrm{Fe}$ layer in $\mathrm{Cu} / \mathrm{Fe}$ sample than that in $\mathrm{Ta} / \mathrm{Fe}$ sample. To directly show the surface roughness of the Fe layers, we characterized the crosssections of the samples by HRTEM. The TEM specimens with cross-sections were extracted from the macroscopic samples using focus ion beam (FIB). Figures 1(a) and 1(b) show the bright-field HRTEM images of the cross-sections of selected samples, $\mathrm{Ta} / \mathrm{Fe}$ and $\mathrm{Cu} / \mathrm{Fe}$, respectively. The dark areas could be easily identified as Fe layers and the thickness is consistent with the preset value of $5 \mathrm{~nm}$. The bottom $\mathrm{SiO}_{2}$ layers are from the thermally oxidized wafer substrates and the top $\mathrm{SiO}_{2}$ layers were deposited by sputtering. The 0.2-nm-thick seed layers, $\mathrm{Ta}$ or $\mathrm{Cu}$, between $\mathrm{Fe}$ and substrates can hardly be identified. As seen in Figs. 1(a) and 1(b), the thickness of Fe layers is identical to each other, but the interfaces between top $\mathrm{SiO}_{2}$ and $\mathrm{Fe}$ layers is much rougher in sample $\mathrm{Cu} / \mathrm{Fe}$ than that in sample $\mathrm{Ta} / \mathrm{Fe}$. The seed layers indeed play an important role on the surface roughness of Fe layers due to the variation of wetting property of Fe on the seed layer and (maybe) also of

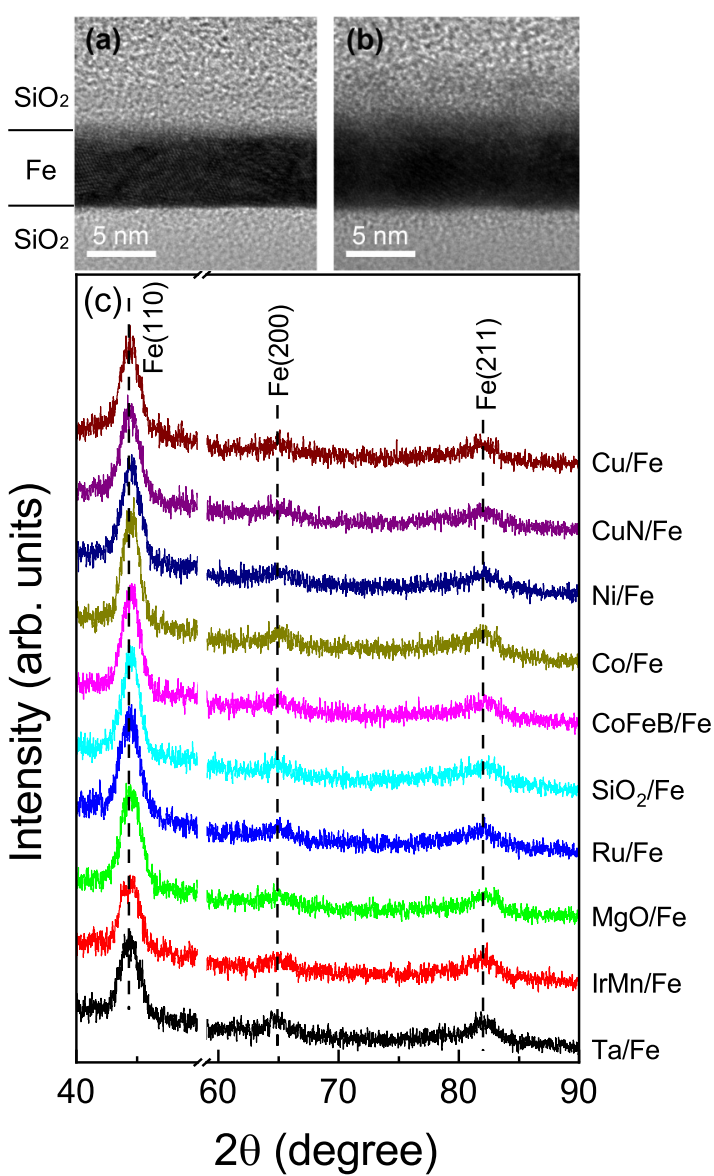

FIG. 1. HRTEM images of cross-sections of the samples (a) $\mathrm{Ta} / \mathrm{Fe}$ and (b) $\mathrm{Cu} / \mathrm{Fe}$. (c) GID spectra for all samples.

the wetting property of seed layers on $\mathrm{SiO}_{2}$. To examine the microstructures of the samples, GID measurements were run for all samples with grazing incident angle of $0.5^{\circ}$. Figure $1(\mathrm{c})$ shows the GID spectra for all samples. The diffraction peaks (110), (200), and (211) of bcc-Fe could be identified. For each peak, the intensity and full width at half maximum (FWHM) are quite identical for all samples, indicating that the samples have similar crystallinity.

\section{B. Longitudinal resistivity}

To understand the surface roughness effect on the electrical transport properties, we measured the temperature-dependent longitudinal resistivity of the samples at zero magnetic field in the temperature range of 5-300 K. Figure 2(a) presents the $\rho_{x x}-T$ curves for all samples. It is evident that all curves show an overall metallic behavior, i.e., all samples have positive temperature coefficient of resistivity (TCR, $d \rho_{x x} / d T$ ) at high temperatures. The upturn of $\rho_{x x}(T)$ curves below very low temperature have a negative TCR and could be ascribed to an electrical conduction dominated by two-dimensional weak localization effect which was verified by the linear dependence of $\rho_{x x}$ on $\ln T$ (see Fig. S2 in Ref. [15]). The most important feature is that the $\rho_{x x}(T)$ curves shift to higher values over the whole temperature range from sample $\mathrm{Ta} / \mathrm{Fe}$ to $\mathrm{Cu} / \mathrm{Fe}$, although the thickness of $\mathrm{Fe}$ layers is identical 

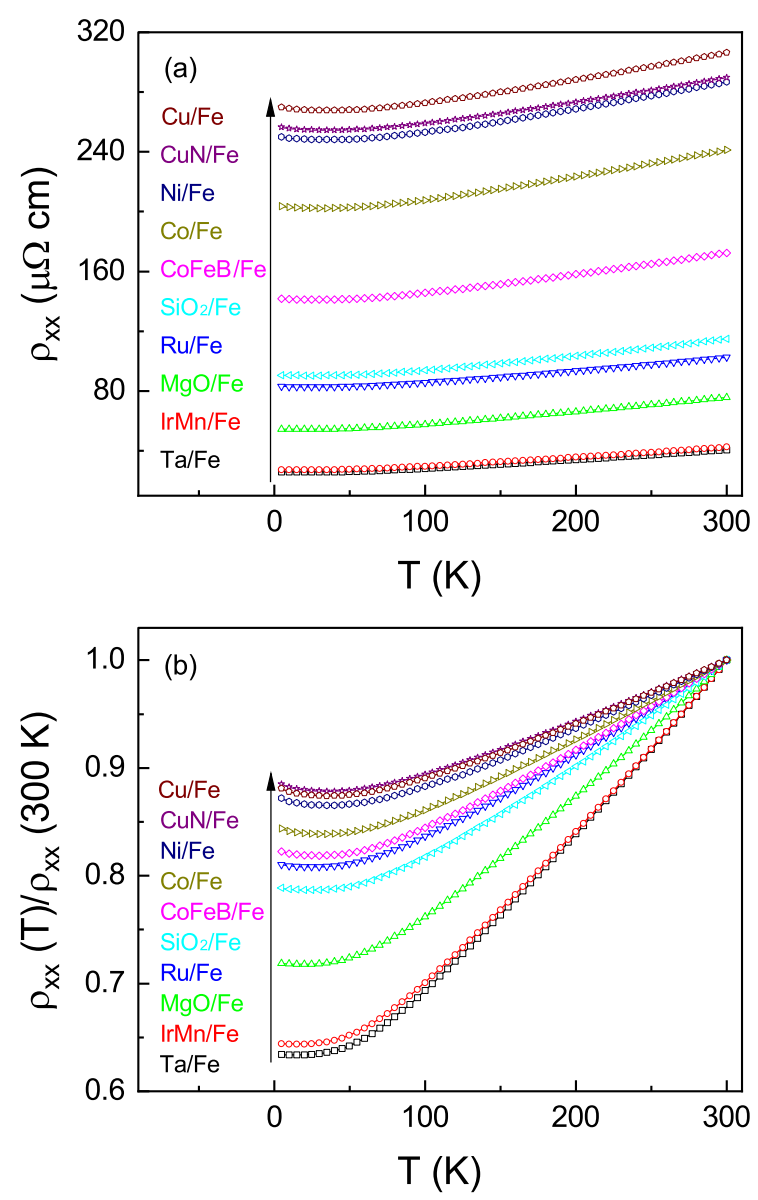

FIG. 2. (a) Temperature-dependent longitudinal resistivity $\left(\rho_{x x}\right)$ curves for all samples. (b) Normalized $\rho_{x x}(T) / \rho_{x x}(300 \mathrm{~K}) \sim T$ curves for all samples.
( $5 \mathrm{~nm}$ ) for all samples. At $5 \mathrm{~K}$, the $\rho_{x x}$ increases by one order of magnitude from sample $\mathrm{Ta} / \mathrm{Fe}$ to $\mathrm{Cu} / \mathrm{Fe}$. The insulating seed layers don't contribute any resistance to the measurement results. For metal or alloy seed layers with thickness of $0.2 \mathrm{~nm}$, they would have much higher resistance than that of Fe layers due to the size effect [11]. Furthermore, the 0.2 -nm-thick seed layers can hardly be continuous layers but rather discontinuous clusters. Therefore, the seed layers have negligible contribution to the longitudinal resistivity of the samples. We ascribe the large resistivity variation of the samples to the surface roughness which could be regarded as effective impurity. At locations where the thickness is thinner than the average thickness, the mean free path of electrons was reduced, which increases the resistivity significantly even though the average thickness is the same. To better understand the surface roughness scattering effect on electrical resistivity, we replotted the data in Fig. 2(a) in the form of $\rho_{x x}(T) / \rho_{x x}(300 \mathrm{~K})-T$, as shown in Fig. 2(b). The resistivity ratio, $\rho_{x x}(T) / \rho_{x x}(300 \mathrm{~K})$, increases from sample $\mathrm{Ta} / \mathrm{Fe}$ to $\mathrm{Cu} / \mathrm{Fe}$, indicating larger scattering effect [22]. $T_{\min }$, defined as the temperature where the $\rho_{x x}$ is minimum in each $\rho_{x x}-T$ curve, increases from sample $\mathrm{Ta} / \mathrm{Fe}$ to $\mathrm{Cu} / \mathrm{Fe}$ as shown in Fig. S3 in Ref. [15], clearly demonstrating more frequent scattering. Overall, the surface roughness of the samples plays an
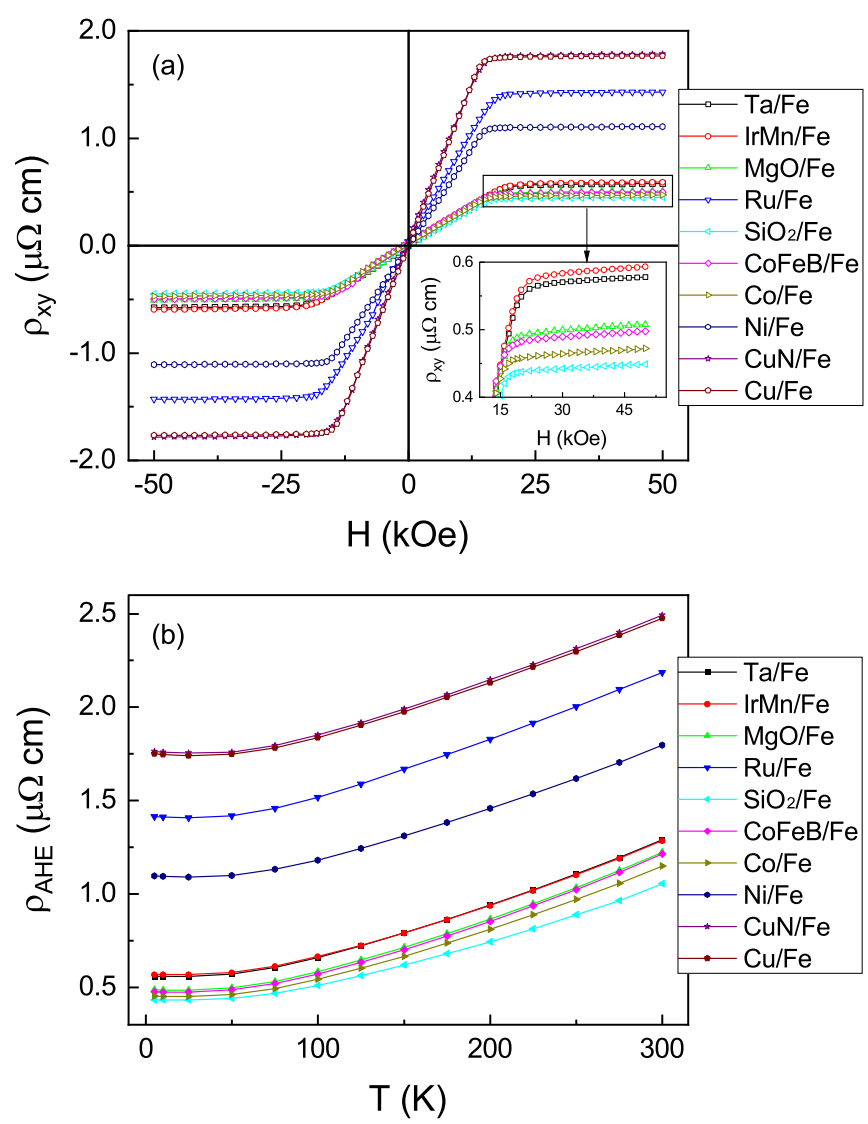

FIG. 3. (a) Field-dependent Hall resistivity $\left(\rho_{x y}\right)$ of all samples measured at 5 K. (b) Temperature-dependent anomalous Hall resistivity $\left(\rho_{\mathrm{AHE}}\right)$ for all samples.

important role in longitudinal resistivity although the samples have the same thickness and microstructure.

\section{Anomalous Hall resistivity}

Since we have observed that the surface roughness significantly affects the longitudinal resistivity, we now turn our attention to that how the surface roughness affects the anomalous Hall resistivity. The Hall resistivity $\left(\rho_{x y}\right)$ for all samples was measured with a magnetic field applied perpendicularly to the plane of the films in a range of $-50 \mathrm{kOe} \leqslant H \leqslant 50 \mathrm{kOe}$ and at temperatures ranging from 5 to $300 \mathrm{~K}$. Figure 3(a) shows the field-dependent Hall resistivity of all samples measured at $5 \mathrm{~K}$. As it is seen, the Hall resistivity follows a linear and strong dependence on applied magnetic field up to the magnetic saturation field $\left(H_{\text {sat }}\right)$. At field higher than $H_{\text {sat }}$, the Hall resistivity shows a linear and much weaker dependence on applied magnetic field. Zero coercivity has been observed in these curves. These are typical behaviors for magnetic thin films having in-plane magnetization at zero field. At high magnetic fields, the weakly field-dependent Hall resistivity should be ascribed to the ordinary Hall effect and the slight change of the AHE due to the improved alignment of spins. At this stage, the field-forced alignment of spins is a result of thermal agitation (except at $0 \mathrm{~K}$ ) and nonferromagnetic coupling at Fe layer surface or defects inside Fe layers. Since Fe has high Curie temperature (1043 K), the thermal agitation 

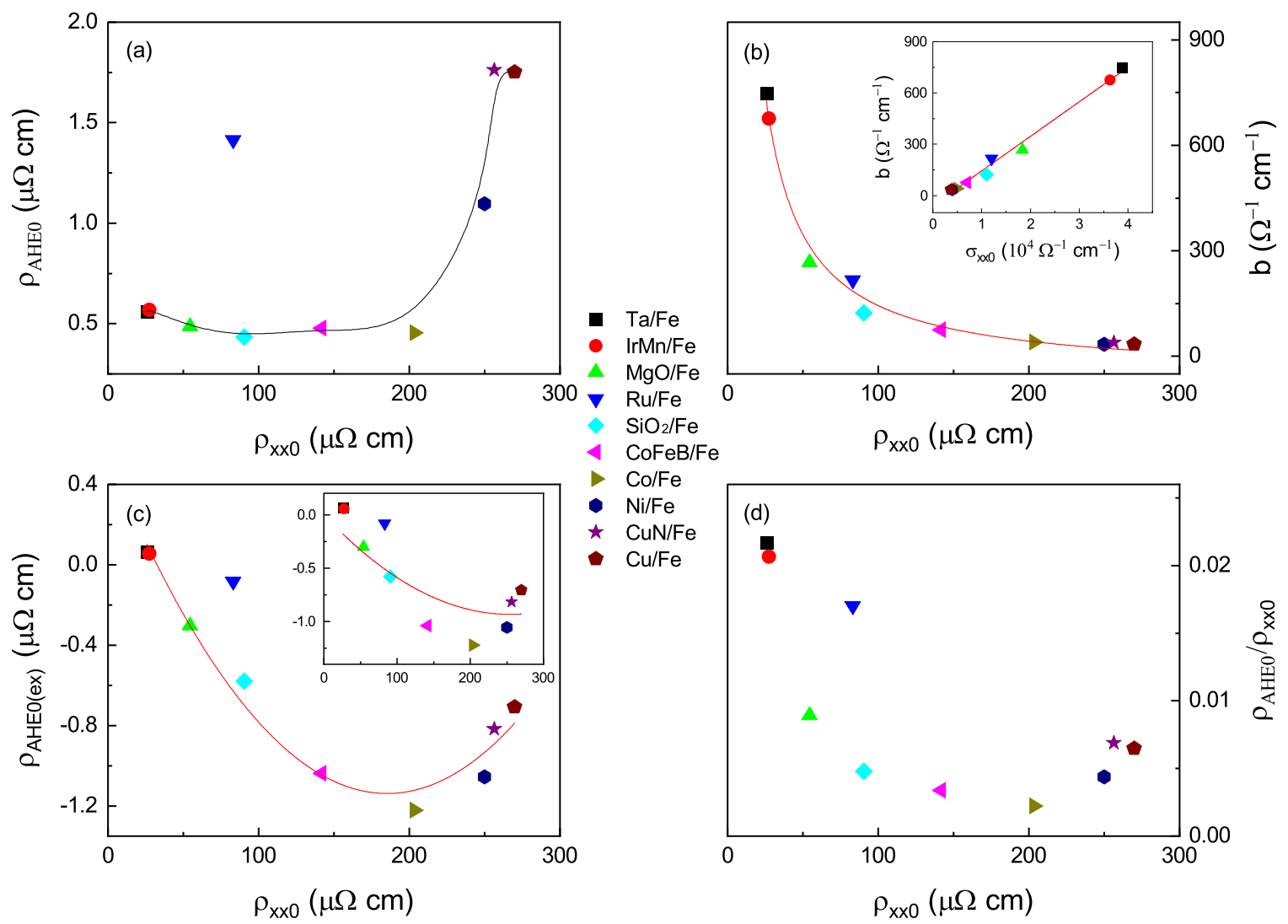

FIG. 4. (a) Anomalous Hall resistivity $\left(\rho_{\mathrm{AHE} 0}\right)$ vs longitudinal resistivity $\left(\rho_{x x 0}\right)$ at $5 \mathrm{~K}$. The solid line is a guide to eyes. (b) Intrinsic anomalous Hall conductivity $(b)$ as a function of $\rho_{x x 0}$. The inset shows the linear dependence of $b$ on $\sigma_{x x 0}$. (c) Extrinsic anomalous Hall resistivity as a function of $\rho_{x x 0}$. The solid line is a fitting curve by Eq. (7). The same set of data shown in the inset was fitted by Eq. (3). (d) The anomalous Hall angle $\left(\rho_{\mathrm{AHE} 0} / \rho_{x x 0}\right)$ at $5 \mathrm{~K}$ vs $\rho_{x x 0}$.

effect could be ignored. For the nonultrathin Fe layers, the nonferromagnetic coupled spins have minor contribution to the Hall resistivity. Therefore, in these samples, the magnitude of anomalous Hall resistivity with negligible error could be obtained by extrapolating the linear part to zero field.

As seen in Fig. 3(a), the Hall resistivity increased by four time from the lowest value to the highest. To better demonstrate the variation of the anomalous Hall resistivity, we plotted the temperature-dependent $\rho_{\mathrm{AHE}}$ for all samples in Fig. 3(b). The sign of $\rho_{\mathrm{AHE}}$ is positive for all samples across the full temperature range, which is consistent with that reported in pure Fe films [9]. The $\rho_{\mathrm{AHE}}-T$ curve for each sample shows the same tendency as $\rho_{x x}-T$ curve. The $\rho_{\text {AHE }}$ flattens off below $50 \mathrm{~K}$ in all samples, indicating that the phonon scattering effect on the AHE could be ignored at low temperatures. The $\rho_{\mathrm{AHE}}$ slightly increases with temperature decreasing at low temperatures, which may be due to the weak localization effect. The slight changes are very small (less than $1 \%$ ) and we could ignore the weak localization effect on $\rho_{\mathrm{AHE}}$ in AHE scaling analysis.

\section{Anomalous Hall effect scaling analysis}

To study the origin(s) of the AHE in these samples, the conventional scaling relation,

$$
\rho_{\mathrm{AHE}} / \rho_{x x}=c+d \rho_{x x},
$$

was usually employed, where $c$ is the contribution of skew scattering, $d$ is the contribution of intrinsic mechanism or side jump. This equation includes both the impurity scattering and phonon scattering effect on mechanisms of skew scattering or side jump. Recently, a new scaling relation [9],

$$
\rho_{\mathrm{AHE}}=\alpha \rho_{x x 0}+\beta \rho_{x x 0}^{2}+b \rho_{x x}^{2},
$$

was proposed in Fe thin films, where $\alpha$ represents the contribution from the skew-scattering, $\beta$ and $b$ denote the sidejump and intrinsic anomalous Hall conductivity (AHC), respectively. The subscript " 0 " indicates that the data were obtained at low temperatures where thermal contribution is negligibly small. This scaling relation excluded the contribution of phonon scattering to skew scattering or side jump. To demonstrate if the phonon scattering affects the extrinsic mechanisms, we plotted the relations, $\rho_{\mathrm{AHE}} / \rho_{x x} \sim \rho_{x x}$ and $\rho_{\mathrm{AHE}} \sim \rho_{x x}^{2}$, for the data of each sample, as suggested in reference [9], to compare the linearity. We found that the relation $\rho_{\mathrm{AHE}} \sim \rho_{x x}^{2}$ shows better linearity for all samples (see Fig. S4 and the corresponding analysis in Ref. [15]). Therefore we could ignore the phonon scattering effect on skew scattering or side jump and use the new scaling, Eq. (2), to analyze our data.

To present the roughness effect on both the longitudinal resistivity and anomalous Hall resistivity, we plotted the relation $\rho_{\mathrm{AHE} 0} \sim \rho_{x x 0}$, obtained at $5 \mathrm{~K}$, for all samples in Fig. 4(a). From sample $\mathrm{Ta} / \mathrm{Fe}$ to $\mathrm{Cu} / \mathrm{Fe}$, the nonmonotonic dependence 
suggests very complicated roughness scattering effect on the AHE. One may ask if the $\rho_{\text {AHE0 }}$ could be affected by the saturation magnetization because anomalous Hall resistivity is not only related to the longitudinal resistivity but also the magnetization $[23,24]$. The saturation magnetization of the samples could be estimated by the magnetic saturation field in Hall resistivity curves, since the samples have in-plane magnetization (demagnetization factor $N=1$ in out-of-plane direction), as shown in Fig. 3(a). The variation of magnetization has minor effect on the $\rho_{\mathrm{AHE}}$.

Since the linear relation, $\rho_{\mathrm{AHE}} \propto \rho_{x x}^{2}$, has been observed for each sample, we could extract the intrinsic AHC which is independent of scattering by Eq. (2). The obtained values of $b$ were plotted as a function of $\rho_{x x 0}$ in Fig. 4(b). The intrinsic AHC dramatically decreases with $\rho_{x x 0}$ increasing, which could be well described by the relation $b \propto 1 / \rho_{x x 0}$ or $b \propto \sigma_{x x 0}$. The inset shows the linear dependence of $b$ on $\sigma_{x x 0}$. Because the $\rho_{x x 0}$ was largely tuned by surface roughness scattering, the relation $b \propto 1 / \rho_{x x 0}$ clearly demonstrates the surface roughness effect on intrinsic AHC. In fact, the electrical transport properties of the samples are largely determined by the areas where the thickness is lower than the average thickness. With thickness decreasing, the intrinsic AHC would decrease, which has been reported in Fe ultrathin films $[9,12]$. For the flattest sample, $\mathrm{Ta} / \mathrm{Fe}$, the intrinsic anomalous Hall conductivity is about $746.7 \Omega^{-1} \mathrm{~cm}^{-1}$ which is very close to the value obtained by first-principles calculation [5].

The intrinsic anomalous Hall resistivity could be subtracted from the total anomalous Hall resistivity and then the extrinsic contribution, including skew-scattering and sidejump, of anomalous Hall resistivity,

$$
\rho_{\mathrm{AHE} 0(\mathrm{ex})}=\alpha \rho_{x x 0}+\beta \rho_{x x 0}^{2}
$$

could be obtained. The data of $\rho_{\text {AHEO(ex) }}$ versus $\rho_{x x 0}$ were plotted in Fig. 4(c). Obviously, this curve cannot be well fitted by Eq. (3). For the scattering-related transport properties in thin films, both the surface scattering and bulk scattering contribute. The longitudinal resistivity at $5 \mathrm{~K}$ could be expressed as

$$
\rho_{x x 0}=\rho_{x x 0(s)}+\rho_{x x 0(b)} .
$$

Accordingly, the scattering-related anomalous Hall resistivity at low temperatures is also expressed as

$$
\rho_{\mathrm{AHE} 0(\mathrm{ex})}=\rho_{\mathrm{AHEO}(\mathrm{s})}+\rho_{\mathrm{AHE} 0(\mathrm{~b})} .
$$

The subscripts $(s)$ and $(b)$ denote the contributions from the surface scattering and bulk scattering, respectively. In Fig. 4(c), the variation of $\rho_{\mathrm{AHEO}(\mathrm{ex})}$ and $\rho_{x x 0}$ is only due to the surface scattering. The bulk scattering contribution, $\rho_{\text {AHEO(b) }}$ and $\rho_{x x 0(b)}$ kept constant for these samples. Therefore using expression

$$
\rho_{\mathrm{AHE} 0(\mathrm{~s})}=\alpha \rho_{x x 0(s)}+\beta \rho_{x x 0(s)}^{2}
$$

instead of Eq. (3) is more appropriate to describe the behavior in Fig. 4(c). If we put Eqs. (4) and (5) into Eq. (6), Eq. (6) could be expressed as

$$
\begin{aligned}
\rho_{\mathrm{AHE}(\mathrm{ex})}= & \left(\alpha-2 \beta \rho_{x x 0(b)}\right) \rho_{x x 0}+\beta \rho_{x x 0}^{2} \\
& +\left(\beta \rho_{x x 0(b)}^{2}-\alpha \rho_{x x 0(b)}+\rho_{\mathrm{AHE}(\mathrm{b})}\right) .
\end{aligned}
$$
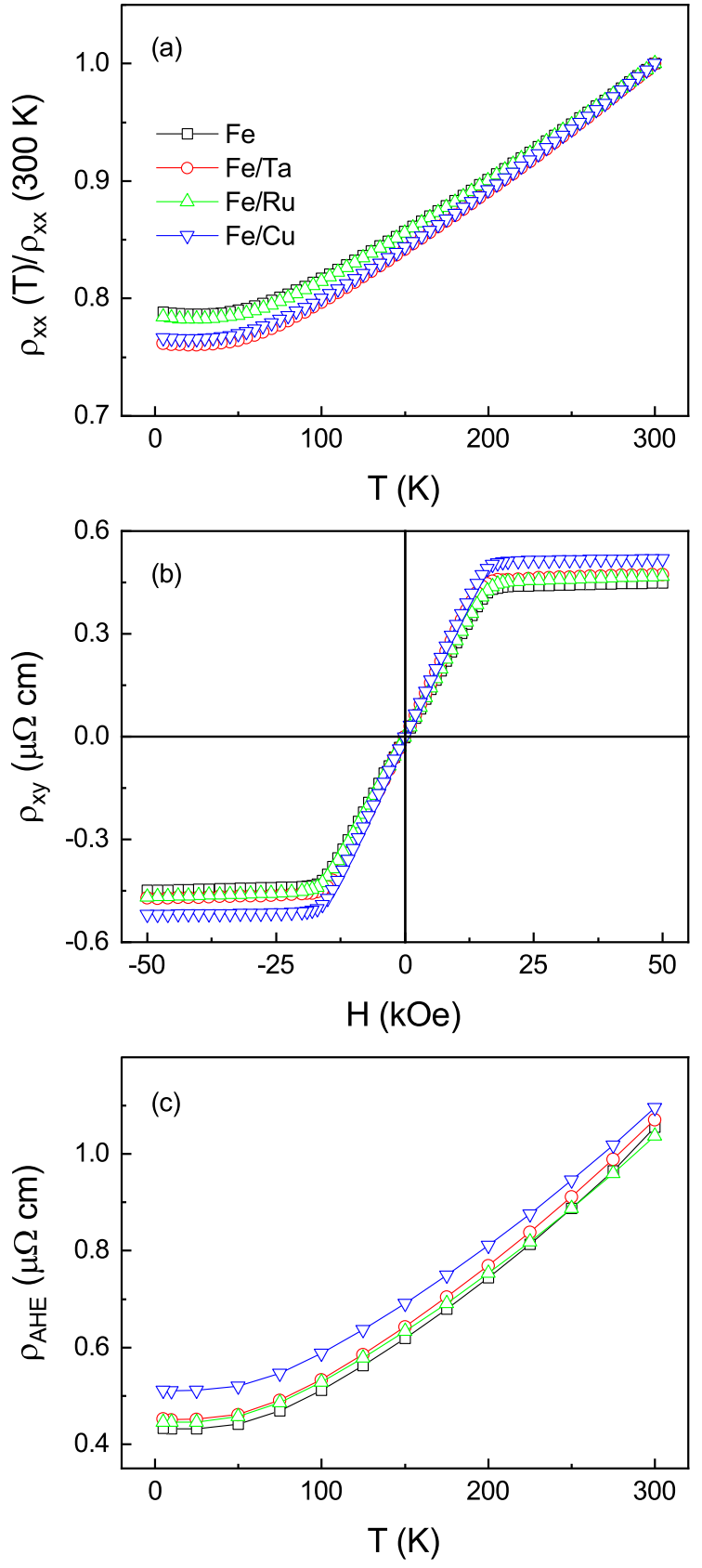

FIG. 5. (a) Normalized $\rho_{x x}(T) / \rho_{x x}(300 \mathrm{~K}) \sim T$ curves for the samples listed in the legend. The legend is the same in (b) and (c). (b) Field-dependent Hall resistivity measured at 5 K. (c) Temperaturedependent $\rho_{\mathrm{AHE}}$ for the samples.

In this equation, $\rho_{\mathrm{AHEO}(\mathrm{ex})}$ and $\rho_{x x 0}$ are variables and other parameters are all constants. Using Eq. (7) to fit the curve in Fig. 4(c), we can extract the parameters as $\beta=$ $40.4 \Omega^{-1} \mathrm{~cm}^{-1}$ and $\alpha-2 \beta \rho_{x x 0(b)}=-1.59 \times 10^{-2}$. Since Eq. (7) is overparametrized, we cannot get the exact value of $\alpha$ but roughly estimate it. For the sample $\mathrm{Ta} / \mathrm{Fe}, \rho_{x x 0}=$ $2.58 \times 10^{-5} \Omega \mathrm{cm}$ and $\rho_{x x 0(b)}$ should be lower than that. Therefore $2 \beta \rho_{x x 0(b)} \ll 1.59 \times 10^{-2}$ and then $\alpha \approx-1.59 \times 10^{-2}$. Comparing the $\rho_{\mathrm{AHEO}(\mathrm{ex})}$ and intrinsic anomalous Hall resistivity, the intrinsic one dominates the anomalous Hall resistivity for all samples. 
Anomalous Hall angle characterized by the ratio of $\rho_{\mathrm{AHE}} / \rho_{x x}$ was also studied for these samples with various surface roughness. Figure 4(d) shows the data, measured at $5 \mathrm{~K}$, of $\rho_{\mathrm{AHE} 0} / \rho_{x x 0}$ versus $\rho_{x x 0}$. The $\rho_{\mathrm{AHE} 0} / \rho_{x x 0}$ decreases with $\rho_{x x 0}$ increases at lower $\rho_{x x 0}$ and increases with $\rho_{x x 0}$ at higher $\rho_{x x 0}$. Overall, the largest anomalous Hall angle was achieved in sample $\mathrm{Ta} / \mathrm{Fe}$, which is the flattest sample with minimum surface roughness. This finding opens a promising avenue for achieving large spin Hall angle by surface roughness engineering in heavy metals.

The previous theoretical study on spin Hall effect has proposed surface roughness enhanced spin Hall angle in $\mathrm{Cu}$ and $\mathrm{Al}$ [17]. In this study, only surface roughness scattering related extrinsic mechanisms were considered and intrinsic mechanism was not discussed. It turns out that the side-jump mechanism contributes to the surface roughness induced spin Hall effect but screw scattering does not. In our work, both screw scattering and side jump contribute to the extrinsic mechanisms which may partially come from the surface scattering, because the roughness scattering and effective surface scattering cannot be separated experimentally. With the surface roughness increases, the side jump contributed anomalous Hall angle $\left(\beta \rho_{x x 0(s)}\right)$ increases, which is consistent with the results of the theoretical work [17].

Since we observed the variation of anomalous Hall resistivity for these samples with different seed layers, one may quest if the variation is caused by the interfacial spin-orbital coupling [25,26] or interfacial modification [27] other than the surface roughness. We then prepared several samples with the same roughness but different interfacial spin-orbital coupling. The sample structure is, from the substrate side, $\mathrm{Fe}(5 \mathrm{~nm}) /$ cover layer $(0.2 \mathrm{~nm}) / \mathrm{SiO}_{2}(5 \mathrm{~nm})$ with different cover layers $(\mathrm{Ta}, \mathrm{Ru}, \mathrm{Cu})$. The sample, $\mathrm{Fe}(5 \mathrm{~nm}) / \mathrm{SiO}_{2}(5 \mathrm{~nm})$, without metal cover layer was also prepared for comparison. The same substrate material $\left(\mathrm{SiO}_{2}\right)$ lead to the same roughness of $\mathrm{Fe}$ layers and different cover layers provide different interfacial spin-orbital coupling. We then measured the temperature-dependent longitudinal resistivity and anomalous
Hall resistivity. Figure 5(a) shows the normalized longitudinal resistivity curves for the four samples. As seen, the resistivity ratio, $\rho_{x x}(T) / \rho_{x x}(300 \mathrm{~K})$, is quite identical, which indicates the similar extent of surface scattering in these samples. Figure 5(b) presents the field-dependent Hall resistivity measured at $5 \mathrm{~K}$ for the four samples. The four curves show similar behaviors and Hall resistivity. The values of $\rho_{\text {AHE }}$, extracted from the Hall resistivity curves at different temperatures, were presented in Fig. 5(c). The $\rho_{\text {AHE }}$ gives identical values at each temperature for different samples, which indicates that the various interfacial spin-orbital coupling cannot explain the large difference of $\rho_{\mathrm{AHE}}$ in Fig. 3(b).

\section{CONCLUSIONS}

We prepared Fe thin films with the same thickness but various surface roughness by using different materials of seed layers. The microstructure and magneto-transport properties were studied systematically. The samples show similar crystallinity as seen in GID patterns and distinct surface roughness confirmed by TEM images of cross-sections. The magnitude of longitudinal resistivity measured at $5 \mathrm{~K}$ increases by one order from sample $\mathrm{Ta} / \mathrm{Fe}$ to $\mathrm{Cu} / \mathrm{Fe}$. The anomalous Hall resistivity at $5 \mathrm{~K}$ vary with different roughness and shows nonmonotonic dependence on $\rho_{x x 0}$. The intrinsic contribution dominated the anomalous Hall resistivity and shows linear dependence on the longitudinal conductivity at $5 \mathrm{~K}$. The anomalous Hall angle depends on the surface roughness, which provides an alternative to achieve large spin Hall effect experimentally.

\section{ACKNOWLEDGMENTS}

The research reported in this publication was supported by funding from King Abdullah University of Science and Technology (KAUST), Office of Sponsored Research (OSR) under Award No. CRF-2017-3427-CRG6. Q.Z. acknowledge the financial support by KAUST sensor project (REP/1/2708$01)$.
[1] E. H. Hall, Philos. Mag. 12, 157 (1881).

[2] R. Karplus and J. M. Luttinger, Phys. Rev. 95, 1154 (1954).

[3] J. W. Ye, Y. B. Kim, A. J. Millis, B. I. Shraiman, P. Majumdar, and Z. Tesanovic, Phys. Rev. Lett. 83, 3737 (1999).

[4] T. Jungwirth, Qian Niu, and A. H. MacDonald, Phys. Rev. Lett. 88, 207208 (2002).

[5] Y. G. Yao, L. Kleinman, A. H. MacDonald, J. Sinova, T. Jungwirth, D. S. Wang, E. G. Wang, and Q. Niu, Phys. Rev. Lett. 92, 037204 (2004).

[6] J. Smit, Physica 21, 877 (1955); 24, 39 (1958).

[7] L. Berger, Phys. Rev. B 2, 4559 (1970).

[8] S. Sangiao, L. Morellon, G. Simon, J. M. De Teresa, J. A. Pardo, J. Arbiol, and M. R. Ibarra, Phys. Rev. B 79, 014431 (2009).

[9] Y. Tian, L. Ye, and X. F. Jin, Phys. Rev. Lett. 103, 087206 (2009).

[10] L. Ye, Y. Tian, X. F. Jin, and D. Xiao, Phys. Rev. B 85, 220403(R) (2012).
[11] D. Z. Hou, Y. F. Li, D. H. Wei, D. Tian, L. Wu, and X. F. Jin, J. Phys.: Condens. Matter 24, 482001 (2012).

[12] L. Wu, K. Zhu, D. Yue, Y. Tian, and X. F. Jin, Phys. Rev. B 93, 214418 (2016).

[13] Z. B. Guo, W. B. Mi, R. O. Aboljadayel, B. Zhang, Q. Zhang, P. G. Barba, A. Manchon, and X. X. Zhang, Phys. Rev. B 86, 104433 (2012).

[14] D. Rosenblatt, M. Karpovski, and A. Gerber, Appl. Phys. Lett. 96, 022512 (2010).

[15] See Supplemental Material at http://link.aps.org/supplemental/ 10.1103/PhysRevB.xx.xxxxxx for the importance of surface/interface roughness in spintronic devices, the definition of surface roughness, AFM analysis of surface roughness for the samples with $\mathrm{SiO}_{2}$ protection layers, two-dimensional weak localization at low temperatures, $T_{\min } \sim \rho_{x x 0}$ relation for all samples, and comparing the linearity of $\rho_{\mathrm{AHE}} / \rho_{x x} \sim \rho_{x x}$ and $\rho_{\mathrm{AHE}} \sim \rho_{x x}^{2}$ for all samples. 
[16] X. H. Wang, J. Xiao, A. Manchon, and S. Maekawa, arXiv:1407.8278v1 [cond-mat.mes-hall].

[17] L. J. Zhou, V. L. Grigoryan, S. Maekawa, X. H. Wang, and J. Xiao, Phys. Rev. B 91, 045407 (2015).

[18] L. Vj, N. P. Kobayashi, M. S. Islam, W. Wu, P. Chaturvedi, N. X. Fang, S. Y. Wang, and R. S. Williams, Nano Lett. 9, 178 (2009).

[19] J. Zhang, C. Liu, Y. H. Shu, and J. Fan, Appl. Surf. Sci. 261, 690 (2012).

[20] F. Ruffino, V. Torrisi, G. Marletta, and M. G. Grimaldi, Appl. Phys. A 100, 7 (2010).

[21] W. F. Shen, D. Mazumdar, X. J. Zou, X. Y. Liu, B. D. Schrag, and G. Xiao, Appl. Phys. Lett. 88, 182508 (2006).
[22] M. Reghu, C. O. Yoon, D. Moses, and A. J. Heeger, in Handbook of Conducting Polymers, edited by T. A. Skotheim, R. L. Elsenbaumer, and J. R. Reynolds (Marcel Dekker, New York, 1996), p. 27.

[23] Q. Zhang, P. Li, Y. Wen, X. He, Y. L. Zhao, J. L. Zhang, and X. X. Zhang, J. Phys. D: Appl. Phys. 50, 235002 (2017).

[24] Q. Zhang, Y. Wen, Y. L. Zhao, P. Li, X. He, J. L. Zhang, Y. He, Y. Peng, R. H. Yu, and X. X. Zhang, J. Phys.: Condens. Matter 29, 415802 (2017).

[25] X. Wang, J. Xiao, A. Manchon, and S. Maekawa, Phys. Rev. B. 87, 081407(R) (2013).

[26] J. Borge, C. Gorini, G. Vignale, and R. Raimondi, Phys. Rev. B 89, 245443 (2014).

[27] J. L. Xu, Y. F. Li, D. Z. Hou, L. Ye, and X. F. Jin, Appl. Phys. Lett. 102, 162401 (2013). 